\title{
OFERTA Y PUBLICIDAD DE ALIMENTOS Y BEBIDAS EN INSTITUCIONES EDUCATIVAS Y ENTORNOS ESCOLARES DE LIMA METROPOLITANA. UN ESTUDIO EXPLORATORIO
}

\author{
Lorena Saavedra-Garcia(1),a, Mayra Meza-Hernández (101,b, Kiomi Yabiku-Soto(1),c, \\ Akram Hernández-Vásquez@1,d, Helena V. Kesar(101,e, Cahuide Mejia-Victorio@1,f, \\ Francisco Diez-Canseco@1,9 \\ 1 CRONICAS, Centro de Excelencia en Enfermedades Crónicas, Universidad Peruana Cayetano Heredia, Lima, Perú. \\ ${ }^{a}$ Licenciada en Nutrición, magíster en Alimentación, Nutrición y Metabolismo, ${ }^{\mathrm{b}}$ licenciada en Nutrición; ${ }^{\mathrm{c}}$ bachiller en \\ Nutrición; ${ }^{\mathrm{d}}$ médico cirujano; magíster en Gestión y Políticas Públicas; ${ }^{\mathrm{e}}$ bachiller en Ciencias Biológicas y Médicas; \\ ${ }^{\mathrm{f}}$ médico cirujano; ${ }^{\mathrm{g}}$ psicólogo, magíster en Salud Pública.
}

\section{RESUMEN}

El objetivo del estudio fue describir la oferta y la publicidad de alimentos y bebidas en instituciones educativas y entornos escolares de 15 colegios públicos y privados de Lima en 2019. Se realizaron observaciones inopinadas y se registraron los alimentos ofrecidos, la presencia de advertencias publicitarias, octógonos y la publicidad en quioscos y cafeterías. A la hora de salida se observó la venta ambulatoria de alimentos. Todas las escuelas ofrecían productos ultraprocesados, el 73,3\% vendía alimentos con octógonos y el $60 \%$ tenía publicidad de alimentos procesados y ultraprocesados. En el $86,7 \%$ de los colegios había vendedores ambulantes de alimentos, varios de los alimentos eran productos ultraprocesados. Los resultados sugieren un ambiente alimentario no saludable dentro y alrededor de las escuelas, y el incumplimiento de la regulación actual. Resulta necesario mejorar la difusión y supervisión de la política alimentaria, y construir con las escuelas estrategias para promover una alimentación saludable.

Palabras clave: Legislación Alimentaria; Alimentos y Bebidas; Publicidad de Alimentos; Alimentos Vendidos en la Vía Pública; Escuelas (Fuente: DeCS BIREME).

\section{FOOD AND BEVERAGE SUPPLY AND ADVERTISING IN SCHOOLS AND THEIR SURROUNDINGS IN METROPOLITAN LIMA. AN EXPLORATORY STUDY}

\begin{abstract}
This study aimed to describe the supply and advertising of foods and beverages both inside educational institutions and in their respective local areas in 15 public and private schools in Lima during 2019. Unbiased observations were recorded regarding the types of food available, the presence of octagons, and advertisements used in kiosks and cafeterias. Mobile food vendors were observed at the end of the school day. All schools offered processed products, $73.3 \%$ sold food with octagons, and $60 \%$ displayed advertising for processed and ultraprocessed foods. Mobile food vendors were observed in $86.7 \%$ of schools, several of which were found to sell ultraprocessed products. The results suggest an unhealthy food environment both inside and outside schools, and a noncompliance with current regulations. It is therefore necessary to improve the dissemination and supervision of the food policy, and develop strategies with schools to promote healthy eating.
\end{abstract}

Keywords: Food Legislation; Food and Beverages; Food Labelling; Schools (Source: MeSH NLM).

\section{INTRODUCCIÓN}

Durante las últimas décadas se ha incrementado en América Latina el consumo de productos procesados y ultraprocesados, caracterizados por tener mayor densidad energética y elevadas concentraciones de sodio, grasas y azúcares añadidos ${ }^{(1)}$. El mayor consumo de estos alimentos ha contribuido al aumento del exceso de peso en niños, niñas y adolescentes que se observa en toda la región, incluido el Perú ${ }^{(2,3)}$. 
Las escuelas son espacios esenciales para la alimentación de niños, niñas y adolescentes. La evidencia de Europa, Norteamérica ${ }^{(4,5)}$ y América Latina ${ }^{(6,7)}$ muestra un incremento en la oferta de alimentos ultraprocesados en instituciones educativas y entornos escolares, haciéndolos más accesibles a los menores.

En el 2013, el Gobierno peruano promulgó la Ley de Promoción de la alimentación saludable para niños, niñas y adolescentes (Ley 30021) ${ }^{(8)}$, que establece el uso de advertencias publicitarias en los empaques de bebidas y alimentos procesados y ultraprocesados para indicar la presencia de grasas trans y cantidades elevadas de azúcar, grasas saturadas y sodio, a la vez que busca regular la publicidad de estos productos y promover la alimentación saludable entre los menores de edad mediante la prohibición de algunas estrategias de mercadeo. El uso de las advertencias entró en vigor recién en el 2019, bajo la forma de octógonos negros (alto en sodio, alto en azucar, alto en grasas saturadas o contiene grasas trans) que alertaban sobre la presencia excesiva de dichos nutrientes críticos. Ese mismo año, el Ministerio de Salud (MINSA) publicó los «Lineamientos para la promoción y protección de la alimentación saludable en las instituciones educativas públicas y privadas de la educación básica», donde se listaban los alimentos recomendados para su venta en quioscos y cafeterías escolares y se indicaba que debían estar libres de octógonos ${ }^{(9)}$.

Para evaluar esta política pública resulta indispensable conocer si las medidas propuestas por el Estado se cumplen al interior de las instituciones. Por otro lado, es relevante conocer el entorno alimentario de las escuelas, como por ejemplo la venta ambulante. Si bien, no existe una regulación para todo el país sobre la venta de alimentos en los entornos escolares, existen compromisos gubernamentales que pueden impulsarla, como el Pacto de Milán, donde ciudades como Lima, se han comprometido a promover dietas más saludables mediante estrategias como normas de comercialización de alimentos y bebidas para niños.

Este estudio exploratorio busca describir la oferta de alimentos naturales, procesados, artesanales y ultraprocesados y la publicidad de alimentos procesados y ultraprocesados, en quioscos y entornos de 15 colegios de Lima Metropolitana, a fines del 2019. Nuestros hallazgos serán útiles para diseñar evaluaciones de mayor envergadura, que permitan valorar el cumplimiento de las regulaciones, identificar barreras y sugerir cambios para garantizar el logro de los ob-

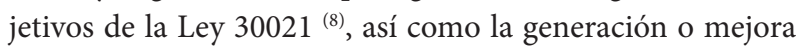
de otras políticas alimentarias que promuevan ambientes alimentarios saludables alrededor de las escuelas.

\section{EL ESTUDIO}

Se realizó un estudio observacional, descriptivo y transversal. Con base en los contactos del equipo de investigación

\section{MENSAJES CLAVE}

Motivación para realizar el estudio: Diversas normas en el país buscan promover la alimentación saludable en escolares; sin embargo, se desconoce la situación de la oferta y la publicidad de los alimentos en los entornos escolares.

Principales hallazgos: Los quioscos de 15 colegios evaluados ofertaban alimentos ultraprocesados; el 73,3\% ofrecía al menos un producto con octógonos y el $60 \%$ tenía publicidad de estos alimentos. En el $86,7 \%$ de los colegios existía venta ambulatoria de alimentos, muchas veces procesados o ultraprocesados.

Implicancias: Es necesario tomar acciones orientadas al cumplimiento de las normas vigentes en escuelas para promover una alimentación saludable en los niños, niñas y adolescentes.

con madres de estudiantes y con docentes, se seleccionaron por conveniencia 15 colegios, 9 públicos y 6 privados, de diez distritos de Lima Metropolitana (Tabla 1).

Entre noviembre y diciembre del 2019, se evaluaron los alimentos ofrecidos y la publicidad de alimentos procesados y ultraprocesados en quioscos y cafeterías escolares, así como la oferta de alimentos por los vendedores ambulantes ubicados, a la hora de salida, a lo largo de la calle frente a las puertas de estas instituciones.

El recojo de información se realizó mediante la observación. Dentro de los colegios, tanto madres como docentes voluntarias, previamente capacitadas, completaron una ficha de observación durante el horario de clases. La observación de la venta ambulatoria la realizó el equipo de investigación, que completó una ficha similar a la salida de los estudiantes. La ficha de observación, diseñada según los lineamientos del MINSA ${ }^{(9)}$, permitía registrar a) las categorías de alimentos vendidos: (I) ultraprocesados, (II) procesados y artesanales, y (III) alimentos naturales; b) la presencia visible de octógonos en estos; y c) las características de la publicidad de alimentos procesados y ultraprocesados (Tabla 2).

Se realizó un análisis univariado de frecuencias relativas y absolutas para describir las categorías de los alimentos ofrecidos en las instituciones educativas y por vendedores ambulantes, la presencia de octógonos en cada categoría y la presencia de publicidad. Los datos se analizaron con Stata 14 (StataCorp, College Station, TX, EUA).

El protocolo fue exonerado de revisión por el Comité Institucional de Ética de la Universidad Peruana Cayetano Heredia (CAREG-ORVEI-158-19).

\section{HALLAZGOS}

Se visitaron 15 instituciones educativas, 60\% públicas y el $73 \%$ con primaria y secundaria, ubicadas en 10 distritos de 
Tabla 1. Características de los colegios incluidos en el estudio.

\begin{tabular}{lllcccc}
\hline Colegio & \multicolumn{1}{c}{ Distrito de ubicación } & Tipo & $\begin{array}{c}\text { Nivel educa- } \\
\text { tivo }\end{array}$ & Alumnos & $\begin{array}{c}\text { Número de quios- } \\
\text { cos/ cafeterías }\end{array}$ & $\begin{array}{c}\text { Número de vendedores } \\
\text { ambulantes }\end{array}$ \\
\hline 1 & Los Olivos & Privado & P, S & 2097 & 1 & 9 \\
2 & Surco & Público & P, S & 671 & 2 & 0 \\
3 & San Isidro & Privado & P, S & 711 & 1 & 1 \\
4 & Villa El Salvador & Público & P, S & 400 & 2 & 7 \\
5 & Miraflores & Privado & P, S & 715 & 1 & 2 \\
6 & Villa El Salvador & Público & P, S & 565 & 1 & 2 \\
7 & Villa María del Triunfo & Público & P & SD & 1 & 5 \\
8 & Surco & Privado & P, S & 1069 & 2 & 3 \\
9 & Cercado de Lima & Público & P, S & 350 & 1 & 1 \\
10 & Pueblo Libre & Público & S & 151 & 1 & 1 \\
11 & Pueblo Libre & Público & S & 181 & 1 & 1 \\
12 & La Molina & Privado & P, S & 735 & 1 & 1 \\
13 & Barranco & Privado & P, S & 340 & 1 & 3 \\
14 & Cercado de Lima & Público & P, S & 470 & 1 & 1 \\
\hline
\end{tabular}

P: primaria; S: secundaria; SD: sin dato.

Lima Metropolitana. Todas tenían al menos un quiosco o cafetería, un $20 \%(\mathrm{n}=3$ ) tenía dos y el número de vendedores ambulantes alrededor del colegio varió desde cero hasta diez (Tabla 1).

Al evaluar la venta de alimentos ultraprocesados en los quioscos (Tabla 3), se observó que todas las instituciones los ofrecían. Los más comunes eran galletas y bizcochos (100\%, $\mathrm{n}=15)$, caramelos y golosinas $(86,7 \%, \mathrm{n}=13)$, bebidas envasadas $(80 \%, \mathrm{n}=12)$ y cereales endulzados $(80 \%, \mathrm{n}=12)$. Además, se observó al menos un producto con octógono(s) en $73,3 \%(n=11)$ de los colegios. Las categorías de alimentos con octógonos más observadas fueron caramelos y golosinas $(53,3 \%, \mathrm{n}=8)$, bebidas envasadas $(53,3 \%, \mathrm{n}=8)$, cereales endulzados $(46,7 \%, \mathrm{n}=7)$ y snacks $(46,7 \%, \mathrm{n}=7)$.

Todos los colegios ofrecían también alimentos procesados y artesanales. Los más comunes eran sándwiches, snac$k s$ derivados de cereales y leguminosas, platos de entrada o principales, y frutas o verduras, encontrados en el 93,3\% de los colegios (Tabla 3 ).

En nueve de los colegios, seis de los cuales eran públicos y tres privados, se observó publicidad de alimentos procesados y ultraprocesados en quioscos o cafeterías. En ocho colegios, cinco públicos y tres privados, se vio publicidad en el mobiliario donde se almacenan los alimentos (congeladoras o estantes); y en dos colegios públicos había afiches publicitarios. Uno de los afiches incluía octógonos y había otros con personajes animados y personajes públicos. Ninguna publicidad ofrecía regalos, premios ni beneficios como la obtención de fuerza, la ganancia o pérdida de peso, o la adquisición de estatus o popularidad. No hubo publicidad que indicara que un producto era «libre de octógonos».

Se contabilizaron 47 vendedores de alimentos en las puertas de salida del $86,7 \%(n=13)$ de colegios visitados. En dos instituciones privadas, no se observaron vendedores ambulantes de alimentos. El promedio fue 3,4 (Desviación estándar - DE: 3,3), y el máximo fue 10 vendedores por colegio. Del total de vendedores, el 61,7\% $(n=29)$ ofrecía alimentos ultraprocesados, y el 34\% ( $\mathrm{n}=16)$ alimentos con octógonos. En los 13 colegios con venta ambulatoria se ofrecía al menos un producto ultraprocesado. Los más observados fueron galletas y bizcochos $(84,6 \%, \mathrm{n}=11)$, cereales endulzados $(84,6 \%$, $\mathrm{n}=11)$, snacks $(69,2 \%, \mathrm{n}=9)$ y caramelos y golosinas $(69,2 \%$, $\mathrm{n}=9)$. En el $84,6 \%(\mathrm{n}=11)$ de los colegios con venta ambulatoria se ofrecían alimentos con octógonos. Las categorías de alimentos con octógonos más observados entre ambulantes fueron galletas y bizcochos $(69,2 \%, \mathrm{n}=9)$, snacks $(61,5 \%$, $\mathrm{n}=8)$ y bebidas envasadas $(53,8 \%, \mathrm{n}=7)$ (Tabla 4$)$.

Finalmente, el 78,7\% de los vendedores ofrecían productos procesados y artesanales. En 13 colegios con venta ambulatoria se ofrecían productos procesados y artesanales. En varios casos se encontraron productos diferentes a los ofrecidos en quioscos y cafeterías, como helados de hielo caseros, queques caseros o gelatina.

\section{DISCUSIÓN}

Se encontró que se ofrecían alimentos procesados o ultraprocesados al interior de todas los colegios públicos y 
Tabla 2. Variables evaluadas en el estudio.

\begin{tabular}{|c|c|c|}
\hline Variables & Categorías & Subcategoría \\
\hline & & Galletas (dulces, saladas y rellenas) y bizcochos. \\
\hline & & Caramelos y golosinas (caramelos, chocolates, toffees). \\
\hline & & Snacks (de queso, tortillas, papitas fritas). \\
\hline & Alimentos ultraprocesados: formulaciones & $\begin{array}{l}\text { Frutos secos envasados (maní dulce, maní salado, mix de frutos secos con sal o } \\
\text { azúcar añadida). }\end{array}$ \\
\hline & mayormente con sustancias extraídas de & Bebidas envasadas (gaseosas, refrescos envasados, jugos y extractos endulzados). \\
\hline & alimentos, derivadas de constituyentes de & Helados industriales (de crema o hielo). \\
\hline & $\begin{array}{l}\text { alimentos o sintetizadas en laboratorio a } \\
\text { partir de materias orgánicas. }\end{array}$ & Cereales endulzados (cereal para desayuno, barras de cereal). \\
\hline \multirow{3}{*}{$\begin{array}{l}\text { Expendio de } \\
\text { alimentos y } \\
\text { presencia de } \\
\text { octógonos }\end{array}$} & & $\begin{array}{l}\text { Yogur y bebidas lácteas aromatizadas y azucaradas (yogur bebible con } \\
\text { saborizantes y azucarados, yogur batido). }\end{array}$ \\
\hline & & Sopas y fideos instantáneos (sopas instantáneas en sobre o vaso). \\
\hline & & Productos cárnicos fritos y embutidos (hamburguesa, nuggets, hot dog). \\
\hline
\end{tabular}

Platos de entrada o principales (preparaciones caseras como causa, chaufa, pollada).

Alimentos procesados y artesanales: Sándwiches (de palta, pollo o queso).

alimentos elaborados de manera artesanal Agua mineral o de mesa, refrescos y jugos (refrescos naturales, chicha morada, o industrial a partir de alimentos en estado jugos de fruta, agua).

natural y que se les ha añadido sustancias de Snacks derivados de cereales y leguminosas (cancha, popcorn, habas). uso culinario.

Frutos secos (maní con pasas, mix de frutos sin sal).

Otros (postres caseros).

Alimentos naturales: alimentos que se encuentran en su estado natural o no han sido sometidos a modificaciones significativas.

Frutas y verduras (frutas enteras o picadas, ensalada de verduras).

Tipo de publicidad.

Presencia de mensaje «libre de octógonos».

de alime

procesados y ultraprocesados

Presencia de octógonos en la publicidad.
Testimonios o imágenes de personajes animados, artistas, deportistas.

Promociones, sorteos, canjes de premios, entrega de regalos.

Beneficios, como obtención de fuerza, energía, habilidades o popularidad. privados, y que en la gran mayoría se ofertaban también a la hora de salida. Entre los productos ofrecidos con mayor frecuencia, dentro y fuera de los colegios, destacaban tanto galletas y bizcochos como las bebidas envasadas, ambos con alta densidad energética. Además, en tres de cada cuatro colegios había productos con octógonos, lo que incumplía los recientes lineamientos del MINSA ${ }^{(9)}$.

Es importante destacar que, en todos los quioscos, los productos procesados se ofrecían junto a productos artesanales y alimentos saludables, como frutas y verduras, bebidas naturales, así como sándwiches, y platos de entrada o principales. Si bien no se registró información sobre la cantidad de productos ofrecidos por categoría ni la acogida que tienen entre los estudiantes, la presencia de alimentos naturales y preparaciones hechas en los colegios ofrece una oportunidad para incentivar su oferta y su consumo. Existen distintas intervenciones que han logrado aumentar el consumo de alimentos saludables en escolares, especialmente de frutas y verduras, mediante intervenciones educativas o su entrega gratuita ${ }^{(10)}$.

Otro hallazgo relevante, considerando la actual legislación, fue la presencia de publicidad de alimentos procesados y ultraprocesados en los colegios visitados, la mayoría públicos. En al menos dos casos, la publicidad incumplía la regulación que, sin ser del todo precisa, prohibía el uso de testimonios de personajes animados y públicos para promover el consumo de estos alimentos ${ }^{(8)}$. Así como la industria publicita los productos procesados, el Estado y los colegios podrían hacerlo con alimentos más saludables. La evidencia sugiere que la exposición a la publicidad de frutas y verduras en instituciones educativas se asocia a una mayor compra y consumo ${ }^{(10)}$. En Perú, la reducción del precio y el aumento de la visibilidad de las frutas, acompañadas de información nutricional, mostraron un mayor consumo en una institución educativa universitaria peruana, que alberga estudiantes de un centro preuniversitario ${ }^{(11)}$. 
Tabla 3. Tipo de alimentos ofertados y presencia de octógonos en quioscos y cafeterías escolares.

\begin{tabular}{|c|c|c|c|}
\hline \multirow[b]{2}{*}{ Tipo de alimentos } & \multirow[b]{2}{*}{ Número de colegios (\%) } & \multicolumn{2}{|c|}{ Tipo de colegio } \\
\hline & & $\begin{array}{c}\text { Público } \\
\text { n (\%) }\end{array}$ & $\begin{array}{c}\text { Privado } \\
\text { n (\%) }\end{array}$ \\
\hline \multicolumn{4}{|l|}{ Ultraprocesados } \\
\hline Galletas y bizcochos & $15(100,0)$ & $9(60,0)$ & $6(40,0)$ \\
\hline Con octógonos & $6(40,0)$ & $3(50,0)$ & $3(50,0)$ \\
\hline Caramelos y golosinas & $13(86,7)$ & $9(69,2)$ & $4(30,8)$ \\
\hline Con octógonos & $8(53,3)$ & $6(75,0)$ & $2(25,0)$ \\
\hline Bebidas envasadas & $12(80,0)$ & $7(58,3)$ & $5(41,7)$ \\
\hline Con octógonos & $8(53,3)$ & $4(50,0)$ & $4(50,0)$ \\
\hline Cereales endulzados & $12(80,0)$ & $7(58,3)$ & $5(41,7)$ \\
\hline Con octógonos & $7(46,7)$ & $4(57,1)$ & $3(42,9)$ \\
\hline Snacks & $10(66,7)$ & $6(60,0)$ & $4(40,0)$ \\
\hline Con octógonos & $7(46,7)$ & $4(57,1)$ & $3(42,9)$ \\
\hline Yogur y bebidas lácteas aromatizadas y endulzadas & $9(60,0)$ & $5(55,6)$ & $4(44,4)$ \\
\hline Con octógonos & $3(20,0)$ & $1(33,3)$ & $2(66,7)$ \\
\hline Frutos secos envasados & $8(53,3)$ & $3(37,5)$ & $5(62,5)$ \\
\hline Con octógonos & $5(33,3)$ & $2(40,0)$ & $3(60,0)$ \\
\hline Helados industriales & $5(33,3)$ & $3(60,0)$ & $2(40,0)$ \\
\hline Con octógonos & $3(20,0)$ & $1(33,3)$ & $2(66,7)$ \\
\hline Sopas y fideos instantáneos & $1(33,3)$ & $0(0)$ & $1(100,0)$ \\
\hline Con octógonos & $1(33,3)$ & $0(0)$ & $1(100,0)$ \\
\hline Productos cárnicos fritos y embutidos & $13(86,7)$ & $7(53,8)$ & $6(46,2)$ \\
\hline \multicolumn{4}{|l|}{ Procesados y artesanales } \\
\hline Sándwiches & $14(93,3)$ & $8(57,1)$ & $6(42,9)$ \\
\hline Snacks derivados de cereales y leguminosas & $14(93,3)$ & $8(57,1)$ & $6(42,9)$ \\
\hline Platos de entrada y principales & $14(93,3)$ & $9(64,3)$ & $5(35,7)$ \\
\hline Agua, refrescos y jugos & $13(86,7)$ & $8(61,5)$ & $5(38,5)$ \\
\hline Frutos secos & $9(60,0)$ & $4(44,4)$ & $5(55,6)$ \\
\hline Otros productos & $7(46,6)$ & $4(57,1)$ & $3(42,9)$ \\
\hline \multicolumn{4}{|l|}{ Naturales } \\
\hline Frutas y/o verduras & $14(93,3)$ & $8(57,1)$ & $6(42,9)$ \\
\hline
\end{tabular}

Los porcentajes son expresados en función a cada categoría (fila).

Nuestros resultados, aunque no son generalizables, sugieren además que la presencia de vendedores ambulantes de alimentos a las afueras de las instituciones podría ser común, y que su oferta combina productos artesanales y una gran variedad de procesados y ultraprocesados con octógonos. El impacto de la venta de alimentos en los entornos escolares en el estado nutricional se ha demostrado en otros países. En el Reino Unido se observó una asociación positiva entre la presencia de comida rápida en el camino entre la escuela y la casa, y el peso de los estudiantes ${ }^{(12)}$. En Nueva Zelanda se encontró que la presencia de vendedores de alimentos alrededor de los colegios puede influir en la calidad de la dieta de los adolescentes ${ }^{(13)}$, mientras que en los Esta- dos Unidos se encontró que los alimentos ofrecidos cerca de los colegios tenían un gran aporte de energía en la dieta de los estudiantes ${ }^{(14)}$.

La presencia de un gran abanico de productos ultraprocesados con octógonos, dentro y fuera de casi todos los colegios visitadas, junto con publicidad, sugieren la exposición de niños, niñas y adolescentes a un ambiente alimentario no saludable, al que la Ley 30021 busca hacer frente ${ }^{(8)}$. Nuestros hallazgos indican que, a finales del 2019, los colegios evaluados no cumplían la regulación vigente. Además, que aun cuando los colegios no ofrecieran ni promocionaran productos con octógonos, los estudiantes los encontrarían a la salida del colegio. Los esfuerzos por regular esta ofer- 
Tabla 4. Tipo de alimentos ofertados y presencia de octógonos en puestos ambulantes frente a los colegios.

\begin{tabular}{|c|c|c|c|}
\hline \multirow[b]{2}{*}{ Tipo de alimentos } & \multirow[b]{2}{*}{ Número de colegios (\%) } & \multicolumn{2}{|c|}{ Tipo de colegio } \\
\hline & & $\begin{array}{l}\text { Público } \\
\text { n (\%) }\end{array}$ & $\begin{array}{c}\text { Privado } \\
\text { n (\%) }\end{array}$ \\
\hline \multicolumn{4}{|l|}{ Ultraprocesados } \\
\hline Galletas y bizcochos & $11(84,6)$ & $5(45,5)$ & $6(54,5)$ \\
\hline Con octógonos & $9(69,2)$ & $5(55,6)$ & $4(44,4)$ \\
\hline Caramelos y golosinas & $9(69,2)$ & $5(55,6)$ & $4(44,4)$ \\
\hline Con octógonos & $4(30,8)$ & $2(50,0)$ & $2(50,0)$ \\
\hline Bebidas envasadas & $8(61,5)$ & $4(50,0)$ & $4(50,0)$ \\
\hline Con octógonos & $7(53,8)$ & $4(57,1)$ & $3(42,9)$ \\
\hline Cereales endulzados & $11(84,6)$ & $5(45,5)$ & $6(54,5)$ \\
\hline Con octógonos & $1(7,7)$ & $0(0)$ & $1(100,0)$ \\
\hline Snacks & $9(69,2)$ & $4(44,4)$ & $5(55,6)$ \\
\hline Con octógonos & $8(61,5)$ & $4(50,0)$ & $4(50,0)$ \\
\hline Yogur y bebidas lácteas aromatizadas y endulzadas & $6(46,2)$ & $3(50,0)$ & $3(50,0)$ \\
\hline Con octógonos & $1(7,7)$ & $0(0)$ & $1(100,0)$ \\
\hline Frutos secos envasados & $3(23,1)$ & $3(100,0)$ & $0(0)$ \\
\hline Con octógonos & $0(0)$ & $0(0)$ & $0(0)$ \\
\hline Helados industriales & $3(23,1)$ & $1(33,3)$ & $2(66,7)$ \\
\hline Con octógonos & $1(7,7)$ & $0(0)$ & $1(100,0)$ \\
\hline Sopas y fideos instantáneos & $0(0)$ & $0(0)$ & $0(0)$ \\
\hline Con octógonos & $0(0)$ & $0(0)$ & $0(0)$ \\
\hline Productos cárnicos fritos y embutidos & $2(15,4)$ & $1(50,0)$ & $1(50,0)$ \\
\hline \multicolumn{4}{|l|}{ Procesados y artesanales } \\
\hline Preparaciones caseras & $3(23,1)$ & $2(66,7)$ & $1(33,3)$ \\
\hline Agua, refrescos y jugos & $4(30,8)$ & $4(100,0)$ & $0(0)$ \\
\hline Snacks derivados de cereales y leguminosas & $5(38,5)$ & $4(80,0)$ & $1(20,0)$ \\
\hline Frutos secos & $2(15,4)$ & $2(100,0)$ & $0(0)$ \\
\hline Sándwiches & $0(0)$ & $0(0)$ & $0(0)$ \\
\hline Otros productos & $8(61,5)$ & $6(75,0)$ & $2(25)$ \\
\hline \multicolumn{4}{|l|}{ Naturales } \\
\hline Frutas y verduras & $4(30,8)$ & $4(100,0)$ & $0(0)$ \\
\hline
\end{tabular}

Los porcentajes son expresados en función a cada categoría (fila).

ta ambulatoria han sido realizados únicamente por algunos gobiernos locales ${ }^{(15-17)}$. Llama la atención que algunos de los colegios evaluados se encuentran dentro de los distritos que poseen este tipo de regulación, por lo observado, aún queda un largo camino para que estas medidas tengan impacto.

Los resultados, aunque parciales, tienes claras implicancias a nivel de políticas. Primero, es necesario difundir la actual regulación en las escuelas públicas y privadas, de manera clara y eficaz. Segundo, se debe establecer un sistema de supervisión de su cumplimiento que se combine con estrategias innovadoras para promover la oferta y el consumo de alimentos saludables. Tercero, la difusión, supervisión y promoción debe incluir a directores y docentes, comerciantes, madres y padres de familia y estudiantes, para que estas acciones se traduzcan en cambios de conducta y que los entornos alimentarios sean sostenibles en el tiempo. Cuarto, es necesaria la fiscalización en los distritos que poseen una normativa sobre la venta ambulante alrededor de las instituciones educativas y el compromiso de otros gobiernos locales para establecer regulaciones que contribuyan a la promoción de ambientes alimentarios saludables en los entornos escolares.

Entre las fortalezas del estudio destacan su carácter oportuno y haber realizado observaciones inopinadas. Las limitaciones incluyen tener una muestra pequeña y por conveniencia, lo que resta robustez y la posibilidad de generalizar los resultados. Por otro lado, las observaciones se realizaron en un solo día, con lo cual no se capturó la variación en la oferta de productos. Además, al completarse el registro de productos según lo que cada observadora veía desde su 
ubicación (ej. fuera del quiosco), es posible que no siempre se registraran todas las categorías de productos disponibles o no se notaran los octógonos de alimentos que estaban fuera del campo visual de la observadora; sin embargo, esta es la oferta y publicidad a la que están expuestos los escolares, muchos de los cuales podrían basar su decisión de compra en los productos que observan y la publicidad o técnicas de marketing visuales empleadas en los mismos empaques ${ }^{(18)}$. Finalmente, las observaciones se limitaron a registrar la presencia de ciertas categorías de productos, sin evaluar la cantidad o diversidad de productos ofrecidos en cada categoría ni las ventas, lo que daría mayor y mejor información sobre la exposición y la aceptación de los alimentos ofertados.

En conclusión, los hallazgos preliminares y la falta de estudios locales invitan a realizar una evaluación de esta política con una muestra más amplia y un recojo de datos más detallado, cuando se retomen las clases escolares presenciales.
Contribuciones de los autores: LSG, MMH, AHV y FDC concibieron y diseñaron el estudio; MMH, KYS, HVK y CMV recolectaron los datos, y AHV los analizó. Todos los autores participaron en la interpretación de los datos, redacción y aprobación del artículo. FDC, LSG y AHV obtuvieron el financiamiento.

Financiamiento: Este trabajo se realizó como parte del estudio auxiliar del Proyecto de Salud Urbana en América Latina (SALURBAL): «Evaluando la implementación y los efectos de las advertencias publicitarias en el etiquetado de los alimentos en Perú: Un estudio de métodos Mixtos». El proyecto SALURBAL es financiado por la iniciativa «Our Planet, Our Health» (grant 205177/Z/16/z) de Wellcome Trust.

Agradecimientos: Los autores agradecen el apoyo de los investigadores de SALURBAL. Para ver la lista de investigadores, visite: https://drexel. edu/lac/salurbal/team/.

Conflictos de interés: AHV es miembro del comité editor de la Revista Peruana de Medicina Experimental y Salud Pública, y no tuvo participación en ninguna etapa del proceso editorial posterior al envío del presente artículo. Los demás autores declaran no tener conflictos de interés.

\section{REFERENCIAS BIBLIOGRÁFICAS}

1. Organización Panamericana dela Salud. Alimentosybebidas ultraprocesados en América Latina: tendencias, efecto sobre la obesidad e implicaciones para las políticas públicas. Washington D.C.: OPS; 2019. Disponible en https://iris. paho.org/bitstream/handle/10665.2/7698/9789275318645_esp.pdf?seque.

2. Corvalan C, Garmendia ML, Jones-Smith J, Lutter CK, Miranda JJ, Pedraza LS, et al. Nutrition status of children in Latin America. Obes Rev. 2017;18 Suppl 2:7-18. doi: 10.1111/obr.12571.

3. Hermoza-Moquillaza VH, Arellano-Sacramento C, Hermoza-Moquillaza RV, Lozano Aguilar VM. Relación entre ingesta de alimentos ultra procesados y los parámetros antropométricos en escolares. Rev Med Hered. 2019;30(2):68-75. doi: 10.20453/rmh.v30i2.3545.

4. Hanning RM, Luan H, Orava TA, Valaitis RF, Jung JKH, Ahmed R. Exploring Student Food Behaviour in Relation to Food Retail over the Time of Implementing Ontario's School Food and Beverage Policy. Int J Environ Res Public Health. 2019;16(14):2563. doi: 10.3390/ ijerph 16142563.

5. Heroux M, Iannotti RJ, Currie D, Pickett W, Janssen I. The food retail environment in school neighborhoods and its relation to lunchtime eating behaviors in youth from three countries. Health Place. 2012;18(6):1240-7. doi: 10.1016/j.healthplace.2012.09.004.

6. Chacon V, Letona P, Villamor E, Barnoya J. Snack food advertising in stores around public schools in Guatemala. Crit Public Health. 2015;25(3):291-8. doi: 10.1080/09581596.2014.953035.

7. Azeredo CM, de Rezende LF, Canella DS, Claro RM, Peres MF, Luiz Odo $\mathrm{C}$, et al. Food environments in schools and in the immediate vicinity are associated with unhealthy food consumption among Brazilian adolescents. Prev Med. 2016;88:73-9. doi: 10.1016/j.ypmed.2016.03.026.

8. Congreso de la República del Perú. Ley N 30021, Ley de Promoción de la alimentación saludable para niños, niñas y adolescentes. Lima: Congreso de la República; 2013. Disponible en https://busquedas.elperuano.pe/normaslegales/decreto-supremo-que-aprueba-el-reglamento-de-la-ley-n-30021-decreto-supremo-n-017-2017-sa-1534348-4/.

9. Ministerio de Salud. Resolución Ministerial № 195-2019-MINSA. Lineamimentos para la promoción y protección de la alimentación saludable en instituciones educativas, públicas y privadas de la educación básica. Lima: MINSA; 2019. Disponible en https://cdn.www.gob.pe/ uploads/document/file/296301/RM_N_195-2019-MINSA.PDF.

10. Evans CE, Christian MS, Cleghorn CL, Greenwood DC, Cade JE. Systematic review and meta-analysis of school-based interventions to improve daily fruit and vegetable intake in children aged 5 to $12 \mathrm{y}$. Am J Clin Nutr. 2012;96(4):889-901. doi: 10.3945/ajcn.111.030270.

11. Cardenas MK, Benziger CP, Pillay TD, Miranda JJ. The effect of changes in visibility and price on fruit purchasing at a university cafeteria in Lima, Peru. Public Health Nutr. 2015;18(15):2742-9. doi: 10.1017/ S1368980014002730.

12. Green MA, Radley D, Lomax N, Morris MA, Griffiths C. Is adolescent body mass index and waist circumference associated with the food environments surrounding schools and homes? A longitudinal analysis. BMC public health. 2018;18(1):482. doi: 10.1186/s12889-018-5383-z.

13. Clark EM, Quigg R, Wong JE, Richards R, Black KE, Skidmore PM. Is the food environment surrounding schools associated with the diet quality of adolescents in Otago, New Zealand?. Health Place. 2014;30:78-85. doi: 10.1016/j.healthplace.2014.08.008.

14. Borradaile KE, Sherman S, Vander Veur SS, McCoy T, Sandoval B, Nachmani J, et al. Snacking in children: the role of urban corner stores. Pediatrics. 2009;124(5):1293-8. doi: 10.1542/peds.2009-0964.

15. Municipalidad Distrital de Villa María del Triunfo. Ordenanza No 214-2016/MVMT. Regulan la Alimentación Saludable - Entornos Saludables en Instituciones Educativas y Alrededores, y declaran de interés distrital la Promoción, Prevención, Control, y Vigilancia de los Alimentos Saludables en las Instituciones Educativas en el Distrito de Villa María. Lima: MVMT; 2016. Disponible en https://busquedas. elperuano.pe/normaslegales/regulan-la-alimentacion-saludable-entornos-saludables-en-i-ordenanza-no-214-2016mvmt-1351534-1/.

16. Municipalidad Distrital de Barranco. Ordenanza No 448-MDB. Ordenanza que regula la alimentación saludable y fomento de entornos saludables en instituciones educativas y alrededores Lima: MDB; 2016. Disponible en https://busquedas.elperuano.pe/normaslegales/ordenanza-que-regula-la-alimentacion-saludable-y-fomento-de-ordenanza-n-448-mdb-1491301-1/.

17. Municipalidad Metropolitana de Lima. Ordenanza $\mathrm{N}^{\circ} 082-\mathrm{MML}$. Ordenanza de Salud y Salubridad Municipal. Lima: MML; 1995. Disponible en: http://www.munlima.gob.pe/images/descargas/licencias-de-funcionamiento/legislacion/22-ORDENANZA-082-MML.pdf.

18. Theben A, Gerards M, Folkvord F. The Effect of Packaging Color and Health Claims on Product Attitude and Buying Intention. Int J Environ Res Public Health. 2020;17(6):1991. doi: 10.3390/ijerph17061991. 